\title{
Multi-material braids for multifunctional laminates: conductive through-thickness reinforcement
}

\author{
Caroline O'Keeffe ${ }^{1}$, Laura Rhian Pickard ${ }^{1}$, Juan $\mathrm{CaO}^{2}$, Giuliano Allegri ${ }^{1}$, Ivana K. Partridge ${ }^{1}$ and Dmitry S. Ivanov ${ }^{1 *}$ (D)
}

\begin{abstract}
Conventional carbon fibre laminates are known to be moderately electrically conductive in-plane, but have a poor through-thickness conductivity. This poses a problem for functionality aspects that are of increasing importance to industry, such as sensing, current collection, inductive/resistive heating, electromagnetic interference (EMI) shielding, etc. This restriction is of course more pronounced for non-conductive composite reinforcements such as glass, organic or natural fibres. Among various solutions to boost through-thickness electrical conductivity, tufting with hybrid micro-braided metal-carbon fibre yarns is one of the most promising. As a well-characterised method of through thickness reinforcement, tufting is easily implementable in a manufacturing environment. The hybridisation of materials in the braid promotes the resilience and integrity of yarns, while integrating metal wires opens up a wide range of multifunctional applications. Many configurations can be produced by varying braid patterns and the constituting yarns/wires. A predictive design tool is therefore necessary to select the right material configuration for the desired functional and structural performance. This paper suggests a fast and robust method for generating finite-element models of the braids, validates the prediction of micro-architecture and electrical conductivity, and demonstrates successful manufacturing of composites enhanced with braided tufts.
\end{abstract}

Keywords: Tufting, Micro-braiding, Functional composites, Electrical conductivity

\section{Introduction}

Hybridisation of polymer composites with metals has been explored in many alternative forms in order to enhance a spectrum of composites properties. Adding an extra-phase is particularly attractive when addressing the characteristic weakness of traditional composite laminates: through-thickness properties. This includes low strength, low toughness, high brittleness, low thermal and electrical conductivities, etc. The insulating properties of the polymer matrix and low transverse conductivities of carbon fibres present a barrier to various useful applications including resistive or induction heating for more efficient processing $[1,2]$, sensing and current

\footnotetext{
* Correspondence: dmitry.ivanov@bristol.ac.uk; http://www.bristol.ac. uk/composites/

${ }^{1}$ Bristol Composites Institute http://www.bristol.ac.uk/composites/ACCIS), University of Bristol, University Walk, Bristol BS8 1TR, UK

Full list of author information is available at the end of the article
}

collection applications [3], lightning strike protection [4, 5], EMI shielding [6, 7], etc. This problem is exacerbated for other common composite reinforcements, such as glass, Kevlar, or natural fibre, which lack electrical conductivity even in the fibre direction.

Lightweight metal additions present an intuitive solution, with materials to date including metal meshes [8], steel fibre woven or non-crimp plies [6, 9, 10], foils [11] resin additives $[6,12,13]$. Several reviews have documented the rapid advancement in such multifunctional materials [14-19]. With respect to through-thickness conductivity, the existing solutions either enhance inplane conductivity without improving through-thickness conductivity or, as in the case of discrete additives, their conductivity is relatively low compared to the continuous reinforcements. Moreover, discrete sub-reinforcements 
often require substantial changes to infusion and consolidation manufacturing practices.

An effective solution for integrating a continuous conductive reinforcement through the thickness of a laminate is by tufting [20, 21] - Fig. 1. Currently, tufting is mainly considered for structural applications and entails the insertion of a thread using a hollow needle that first travels and then retracts along the insertion path. Held in place solely by friction, either between the yarn and preform or the yarn and a sacrificial foam support, a tuft forms a double length of yarn with a loop on the back face of the preform $[22,23]$. Tufts spanning through the laminate thickness substantially increase throughthickness strength and resistance to inter-ply delamination [24-26]. Carbon, glass or polymer yarns are commonly used for this application with carbon providing the additional benefit of increasing through-thickness conductivity [24, 27-29]. Whilst tufting boosts out-of-plane and antidelamination properties of a composite, its insertion distorts preform fibres and, hence, it can negatively affect the in-plane properties $[24,27,30]$. On balance, the throughthickness performance may be beneficial for many applications where the limiting factor is catastrophic delamination rather than in-plane rupture or breakage of fibres.

Thread damage such as rupture, crimping, and filamentation during processing is also a cause for concern [28]. Regarding thread degradation, only a small number of studies focus on thread types and tuft processing optimisation $[28,31,32]$. This study investigates the hybridisation of fine metal wires with carbon fibre tows to enhance both functionality and manufacturability [33, 34]. Metal wires, such as nickel-chrome, copper, titanium or steel, when tufted alone can be easily damaged or broken. Combining the same fine wires with carbon tows ensures continuity of the conductive path and a much higher level of local conductivity than that achievable with carbon fibre alone.

The most effective way of intermingling dissimilar materials in continuous integral threads is via microbraiding. This process utilizes maypole rotary braiding with two sets of yarns moving in opposite serpentine paths [35], replacing one set with the aforementioned fine metal wires. Braided thread manufacturing does not require a mould to deposit the material, instead the interlaced tows and wires are self-supported via tight contacts within the braid. During tufting these multimaterial, hybrid braided threads offer greater robustness with respect to threads consisting of carbon tows only. Successful tufting of carbon fibre preforms with these hybrid braided threads (hereafter "braids") consisting of four $0.1-\mathrm{mm}$ diameter wires and four $1 \mathrm{~K}$ carbon tows have been reported previously. The resultant composite test parts showed a boost in electrical conductivity and structural resilience to subsequent manufacturing stages including handling, infusion and curing [34].

Micro-braiding offers great flexibility in combining materials and tuning tuft properties by selecting braiding patterns, wires, tow and braid densities. This large design space therefore requires parametric tools for the fast assessment of braid characteristics both in terms of susceptibility to damage and of its functional properties. The choice of braid materials and patterns determines the success or failure of the braiding operation (as some patterns result in wire breakage), the feasibility of tufting and, eventually, the efficacy and efficiency of the desired functional performance.
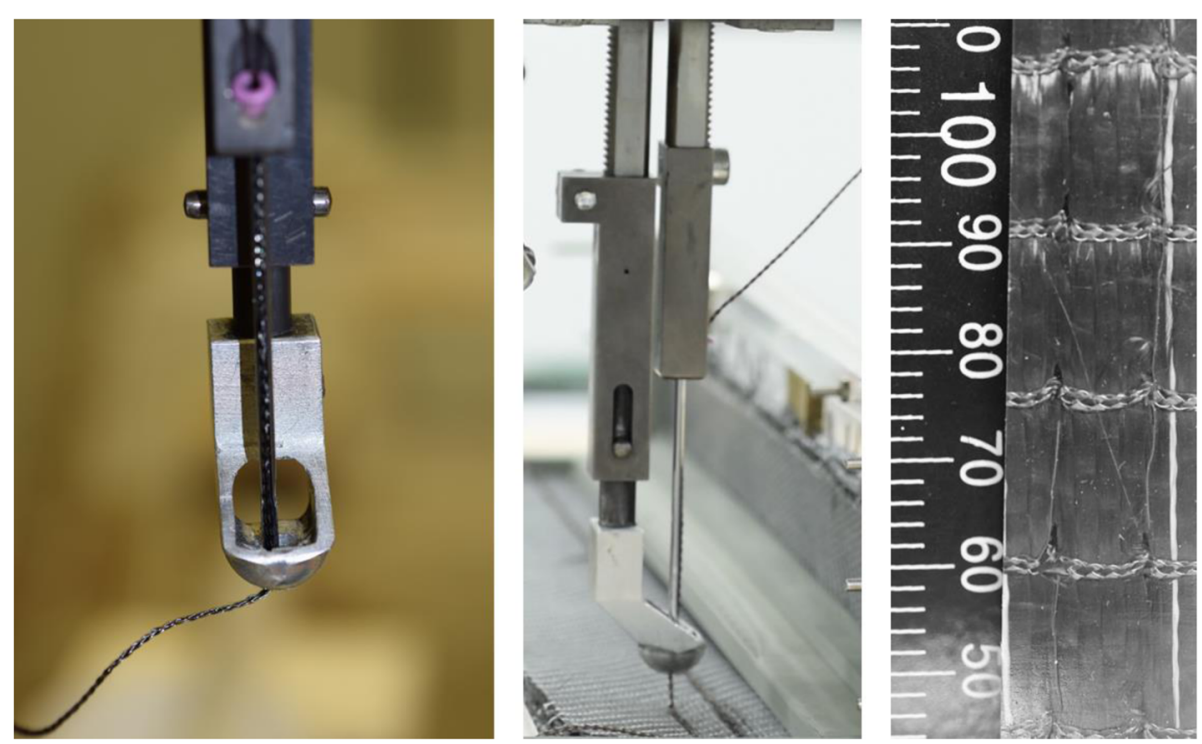

Fig. 1 Tufting needle and thread, tufting into preform tufted preform 
Modelling the braid micro-structure is a complicated task. The architecture of tightly-interlaced components is not trivial and often requires detailed reconstruction of internal geometry [36], computationally-heavy process modelling [37], or excessive simplification in representation of the microstructure. Many of the existing modelling tools for generating complex structures are designed for constructing models of flat preforms and thus not readily available for generating models of microbraids.

This study presents design tools enabling fast and reliable braid model generation. The suggested approach takes as a starting point a geometrical model representing an unrolled braid, as though it were sliced along its length and flattened out. This is constructed as a parametric WiseTex model [38], which allows textile creation for any pattern, with yarn properties defined as an input for the model. WiseTex then calculates an energetically favourable position of the deformable yarns, resulting in a realistic representation of a textile, with each yarn depicted as a series of cross-sections of constant shape along the yarn midline. There is also an option of assigning pre-defined characteristic yarn dimensions. In this study yarns represent deformable carbon fibre tows and defined cross section metal wires. Once ready, the textile model can be converted to a solid model by removing some inconsistencies associated with the simplified geometry and connecting yarn crosssections along the yarn path [39].

Every point on each yarn surface in the flat textile model is assigned a geometrical transformation, which rolls the flat geometry into a tubular shape, representative of the braid. The local fibre orientations follow this same transformation. Thus, as opposed to natural sequence of dealing with braided sleeves (braiding on cylindrical shape, then cutting it open and flattening the sleeve out if a non-tubular structure is to be made) an opposite approach is realised in modelling space - first, making a good approximation of a flat fabric and then revolving the crimped interlaced tows and wires about a central line to produce a consistent micro-braid model. Such an approach is very effective computationally and produces a reasonably realistic representation of the braid architecture.

Once this rolled, solid model is ready and assembled, the model is meshed to produce a voxel-like representation of the textile geometry. An electrical conduction problem is then solved to calculate the effective electrical conductivities of the thread. The results of the simulations are then validated against resistivity measurements of the thread itself and thread integrated into the tufted carbon preform. The latter presents a challenge as the location of measurement makes a significant difference to the measured signal. Hence, careful extraction of one tufted loop was undertaken to carry out these measurements. CT-scans of individual threads also provide important insight on the changes in material properties during tufting.

\section{Experimental characterisation of micro-braided preforms}

Microbraid manufacturing

Multifunctional carbon-metal braids were produced using a commercially available circular maypole 16 horn gear micro-braider Herzog 1/16 80. Various braided patterns, braid densities, thread number (up to 16) and material combinations could be manufactured using the machine. Preliminary manufacturing trials narrowed down the design parameters and, in relation to subsequent tufting process, the following were chosen for optimal performance:

a) Carbon Toray ${ }^{\circ} \mathrm{T} 3001 \mathrm{~K} 67$ tex untwisted tows were trialled with different $0.1 \mathrm{~mm}$ diameter wires including Block NC0100 nickel-chrome, Block CUL 100 copper, and Block titanium wire. Owing to superior electrical conductivity, the discussion is here focused on carbon-copper braids though other metal wires can be likewise efficiently braided.

b) A total of eight yarns (four carbon tows and four metal wires) are used in braid construction. Braids comprising of 16 yarns were also trialled but were found to be too large to fit the tufting needle. The even combination of carbon tow and metal wires was found to ensure a stable braiding process and avoided kinking and twisting along the braid length. This reduced the likelihood of the thread jamming in the tufting needle eyelet causing breakage.

c) The braid pattern was restricted to a diamond interlacement pattern due to the braider operating at half capacity. It was found out that directing carbon and metal in opposite braiding directions resulted in a stable process, whereas placing metal wires in both braiding directions led to breakage of carbon fibre tows. An important feature of the selected configuration is that each of the four wires are separated by carbon yarns and run in parallel to each other.

d) The braid was produced with lay length of $8 \mathrm{~mm}$. At this setting, the braider operated with an up-take speed of $22 \pm 0.9 \mathrm{~m} / \mathrm{h}$ and horn gear rotary speed of $120 \pm 5 \mathrm{rpm}$. Based on image analysis of the resultant braids - Fig. 2 - the braiding angle was determined to range between $10^{\circ}$ and $15^{\circ}$ to the thread direction.

\section{Electrical conductivity of micro-braided threads}

Braid electrical resistance was measured during tensile testing using a Keithley Model 2100 USB Digital 
)

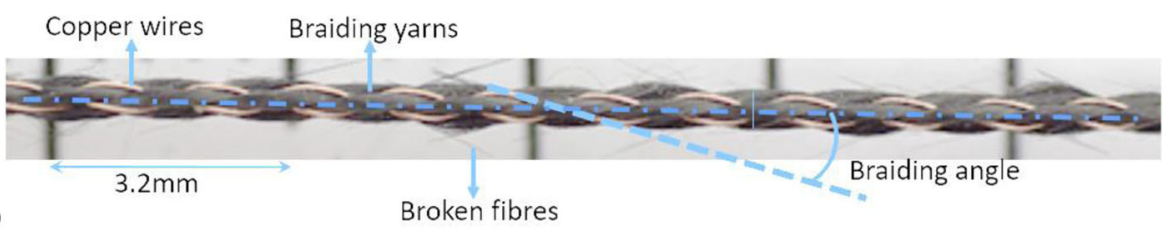

b)

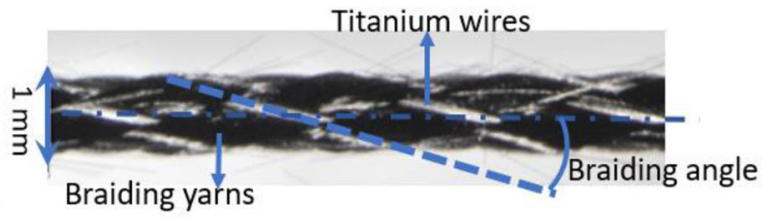

Fig. 2 Example of micro-braided a) carbon-copper, b) carbon-titanium threads

Multimeter. The resistance measurement was undertaken using the four-wire method thereby reducing measurement error by eliminating the voltage drop caused by the leads. Data acquisition was achieved using National Instruments ${ }^{\mathrm{Tm}}$ LabVIEW software which synchronised the recording from the multimeter with the Shimadzu universal testing machine using a National Instruments $^{\text {TM }}$ analogue reader. Initial mechanical testing was carried out according to the British Standard on yarn testing BS EN ISO 2062:2009 [40]. Table 1 provides details of the braid's properties according to this standard. A deviation in the test methodology was made to accommodate measurement of the electrical resistance [40]. Threads of gage length $500 \pm 2 \mathrm{~mm}$ were examined at a testing rate of $25 \mathrm{~mm} / \mathrm{min}$. Initial electrical readings were made at a set pretension raging between 2 and $3 \mathrm{~N}$ according to yarn linear density standard as defined by the standard BS 7658-2: 1993, ISO 10120:1991 [41]. Samples were positioned and tested in a Shimadzu AGSXSSm-DAL-10 kN testing machine using a $1 \mathrm{kN}$ load cell and Shimadzo mechanical radiused clamps. To protect the thread within the grips and to insulate from the metal parts of the instrument, tape was wrapped around affected sections of the thread. To measure the braid resistance, electrodes were attached, such that the internal voltage readings were $20 \mathrm{~cm}$ apart. LabVIEW software synchronized reading from the multimeter with the Shimadzu universal testing machine using a National Instruments analogue reader for data acquisition. Measurements were made at one reading per second.

Table 1 Basic properties of hybrid carbon-copper braid

\begin{tabular}{lll}
\hline & Mean value & Coefficient of variation, \% \\
\hline Breaking force & $94 \mathrm{~N}$ & 9 \\
Extension at break & $8 \mathrm{~mm}$ & 7 \\
Elongation at break & $2 \%$ & 7 \\
Linear density & 533 tex & \\
Breaking tenacity & $18 \mathrm{cN} /$ tex & \\
\hline
\end{tabular}

The mean electrical resistance measurement of the copper carbon braid was $0.20 \pm 0.10 \Omega$ when under pretension sufficient to straighten the braid but without applying considerable deformation. High levels of noise in these initial readings were observed due to the high conductivity of the metal components and the sensitivity limitation of the multimeter. It was deemed plausible that not all metal wires within the braid were engaged in contact with the current collection clips in all the measurements.

Under tensile testing, an increase in electrical resistance was observed as the braid condition decreased during failure. Figure 3 shows a representative response of the normalized electrical resistance of the braid under testing. A typical profile demonstrates three distinct steps. The first is the initial loading where the resistance remains relatively constant. As load is applied, resistance then decreases- often to below the multimeter measurement threshold. This decrease can be attributed to alignment of the carbon fibres and the wires due to the applied tension, which also causes a decrease in braid diameter. This tightening of the braid structure is reasonably expected to increase the number and contact cross section of electrical connections along the length of the braid. Other studies investigating the electrical resistance of carbon fibre z-pin and tufts have also experience a similar response due to initial loading [3, 30].

For the sample with minimum normalized resistance (hence maximum conductivity, suggesting better than average contact between clips and wires within the braid), the scatter in signal acquisition was measured over time (Fig. 3). In this instance the scatter is associated with fluctuation due to measurement noise. These results yielded a resistance of $0.08 \pm 0.02 \Omega$ prior to load application (step 1). During the tensile test, the resistance initially drops below the multimeter measurement threshold as mentioned above (step 2). As the braid fails its resistance ramps to $0.31 \pm 0.05 \Omega$ (step 3). 


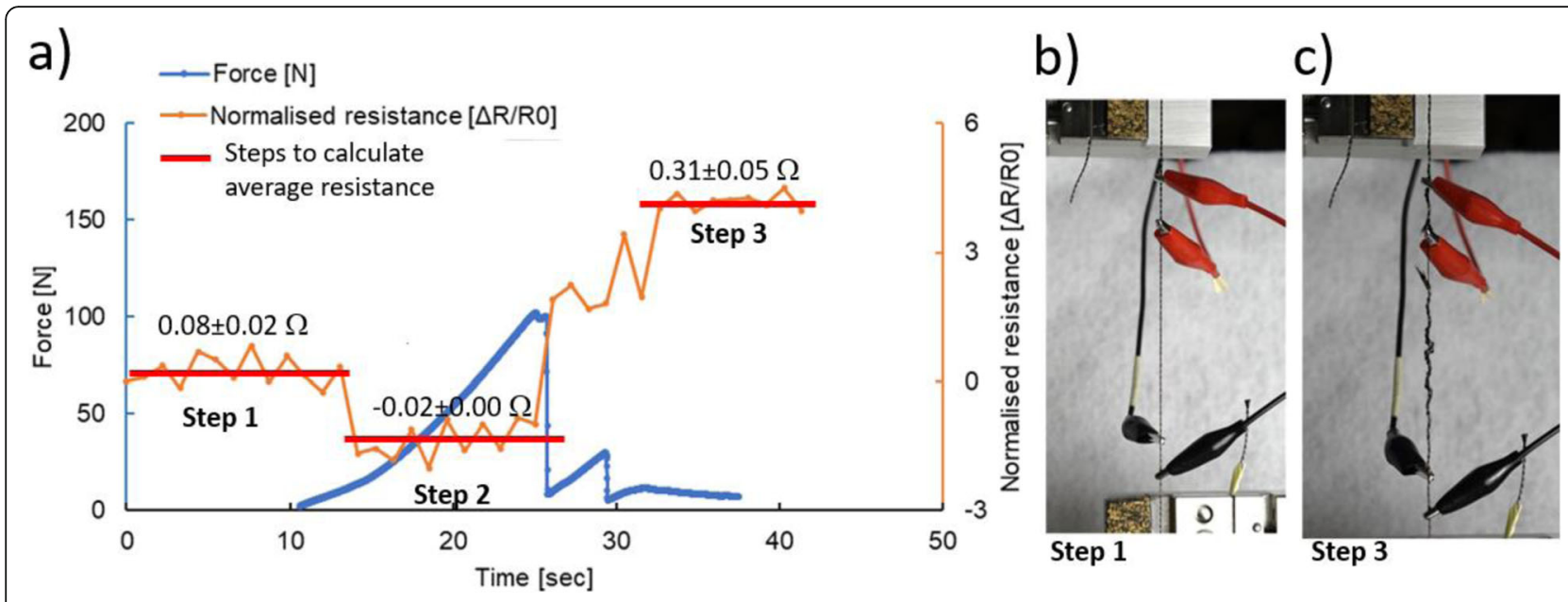

Fig. 3 a Characteristic resistance and load response of hybrid braid in tension, $\mathbf{b}-\mathbf{c}$ images of the braid at initial (under tension) and final (failure) stages of testing

\section{Manufacturing of tufted composites}

Dry carbon preforms consisting of unidirectional (UD) or triaxial quasi-istropic (QI) configurations were laid up by hand. The QI preform consisted of 3 layers of unidirectional carbon fibre in $[0,45,90]$ configuration held together by stitching and binder. The UD fibre plies were each single layer, held together by stitching. Each ply measured $200 \times 200 \mathrm{~mm}$ (QI) or $200 \times 180 \mathrm{~mm}$ (UD). The pre-forms, consisting of 8 sets of 3 plies QI or 24 plies UD were placed on a tufting rig as described above, held in place with cork strips and manual pressure.

An adapted 3D printed chassis with an integrated Schmetz EP $11 \mathrm{Nm} 230$ tufting needle was used to insert tufts in the preform. The diameter of the tufting needle eye was a $1.0 \mathrm{~mm}$ and commercially available off-theshelf. Tufts were inserted vertically at right angles to the preform and held in place in an Airex ${ }^{\circ}$ R6380 foam 25 thick backing, covered with Wrightlen PTFE to prevent contamination. No tension was applied to the braid during the tufting procedure (Fig. 4).

Each panel was tufted in straight lines with $10 \mathrm{~mm}$ distance between the tuft insertions and $10 \mathrm{~mm}$ distance between the tuft rows. Tufts were placed with loops which protruded through the rear of the panel, and loops were arranged such that they did not cross. Preforms were infused using a double bagging vacuum resin infusion method with Prime $27^{\mathrm{m}}$ two-part epoxy resin. Infusion was carried out at room temperature followed by a 2 -h oven cure at $80^{\circ} \mathrm{C}$.

To investigate the internal architecture of the tufted composite, a CT scan on isolated blocks cut from a tufted composite panel were conducted, with the intention of aiming at capturing the through-thickness architecture of an individual tuft. Scans were taken using a Nikon XTEK $320 \mathrm{H}$ platform with a refection target mounted using a flat panel detector. Images were acquired at a voltage of $124 \mathrm{kV}, 142$ micro amps exposed for $500 \mathrm{~ms}$. Each scan was 2600 projections with an average frame rate of 4 , a resolution of $88 \mu \mathrm{m}$. No filter was used. The scan, shows the metal wire only, revealing damage to the wires from tuft insertion - Fig. 5. The scan is a section of a larger panel comprising 24 plies of Formax FCIM359-PB biaxial non-crimp carbon fibre fabric (NCF), containing $24 \mathrm{k}$ HTS carbon fibre tows held together by non-structural stitching.

\section{Through-thickness electrical conductivity of the resulting composite}

To assess the impact of tufting with hybrid braids on the through-thickness conductivity of the final composite, both tufted and untufted unidirectional panels were cut into small blocks 10 by $10 \mathrm{~mm}$ in the plane of the panel (sides aligned with the fibre direction). To eliminate surface roughness prior to measurements, the top and bottom surface were polished subsequently with 400-grit, 800 -grit and 1200-grit sandpaper. Multimeter wires were connected to copper current collection foil, which was attached to the sample surface by manual silver painting. Electrical contact was ensured by applying light compressive stress to the foil using a plexiglass tool. The resistance of foil and the silver painting are of several orders of magnitude lower than the resistance of the material and are ignored in the results processing.

Conductivity was measured three times for each sample. Based on six samples from the untufted panel, the baseline through-thickness conductivity was measured to be $0.74 \pm 0.07 \mathrm{~S} / \mathrm{m}$. Similar measurements for the tufted panel resulted in a large amount of scatter and strongly depended on the location from where the block was cut. The results over 11 samples range in measured 
a)

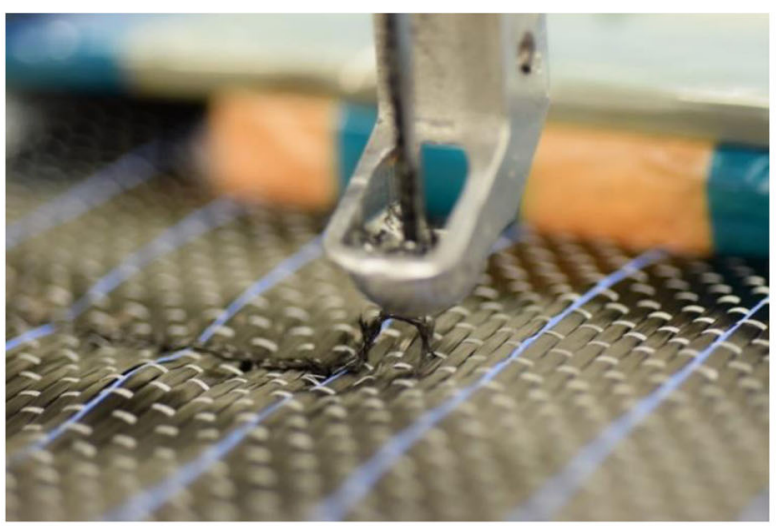

b)

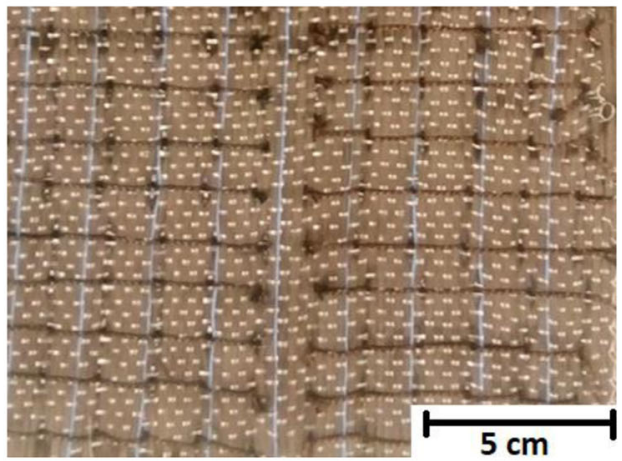

c)

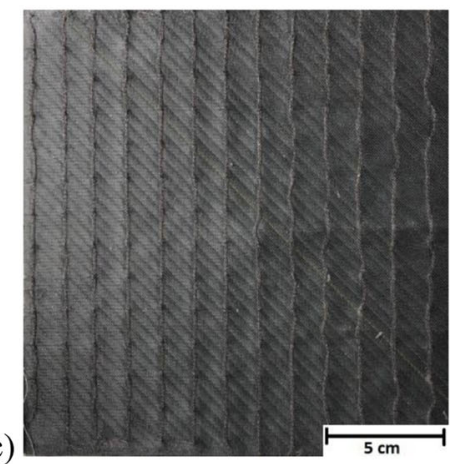

Fig. 4 a Close up of tufting needle showing braid being tufted into a unidirectional preform. $\mathbf{b}$ A tufted unidirectional carbon fibre preform with two sets of braids which contain different materials, c An infused and cured quasi-isotropic carbon fibre-epoxy (Prime 27) panel tufted with carbon-copper braids

values from $0.93 \mathrm{~S} / \mathrm{m}$ to $98.89 \mathrm{~S} / \mathrm{m}$. The samples tested were taken from a range of positions on the tufted panel and do not all contain complete tufts. However, all show at least some improvement over the baseline conductivity. The high variation in electrical conductivity is not caused by the inconsistency of the technology but by the spatial variation of electrical conductivity. Depending on the exact location of the tuft in relation to the small sample extracted from the material the electrical conductivity may be drastically different. In addition, the percolation of the tuft to the surface may be impeded by the presence of epoxy not fully eliminated through polishing. This feature may not compromise the functional performance of the part but does show certain difficulties in characterizing the electrical conductivity of the material system.

The absolute values of the through-thickness conductivity may appear to be low compared to other methods of functionalization [42]. However, it is important to note that for continuously tufted material the electrical conductivity is strongly dependent on the scale of considerations and the architecture of a particular examined volume. Conductivity in the proximity and at the scale of the tufts should be expected to compare to the properties of the metal whereas away from the tuft insertion location is approaching the nominal values of untufted material. Hence, the conductivity may need to be considered as a system property rather than a material property.

\section{Modelling of functional performance of the braid Geometrical modelling of the braid}

The complexity and versatility of microbraid architectures demands a modelling tool that can seamlessly use the parameters of braiding and properties of selected materials to estimate the effective properties of the resulting braid. This can be used to inform the tufting process and enable fast screening of various designs. The purpose of the tool presented here is to do this via fast generation of finite-element models.

The model of a 'slit and unrolled' braid is constructed in fabric modelling software, such as WiseTex [38]. The software generates a model of the fabric based on the input pattern, characteristic yarn dimensions, yarn spacing, 


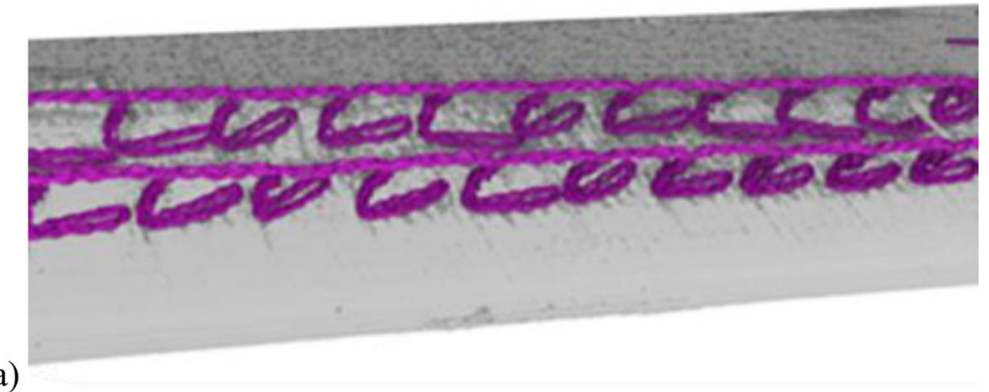

\section{Through-thickness tuft insertion}

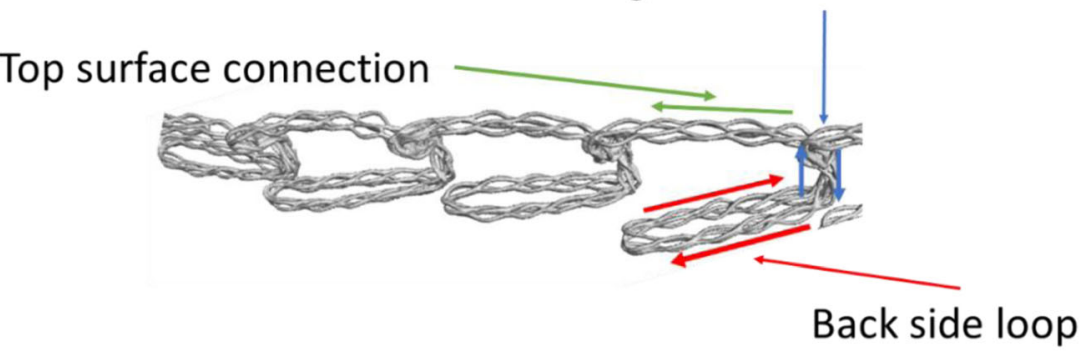

b)

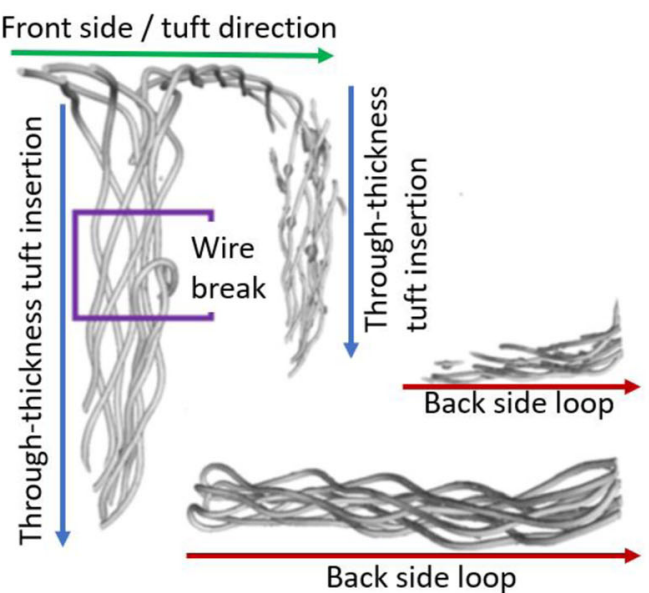

Fig. 5 CT scan highlighting path of metal wire in tufted composite. a two rows of tufts (metal is highlighted in pink colour), b closer look at neighbouring insertion of a tuft, $\mathbf{c}$ higher resolution of a single tuft insertion

and braiding angle. Geometry is approximated by a series of constant-shape cross-sections, whose dimensions, orientations, and positions are calculated based on minimisation of the energy of interacting carbon fibre tows and the set dimensions of the metal wires. Such models of flattened braids can then be converted to finite-element models of the 'rolled' braids by geometrical operations with fine consistency and good quality meshes [39].

In the current work the WiseTex model is used as a starting point to create cylindrical braid geometries - Fig. 6a. The four carbon tow and four copper wire geometry and braiding pattern discussed in the experimental section is represented here.

Prior to mesh conversions the cross-sections of yarns are discretised on a number of contour points (in line with the approach discussed in details in [39], selected to be 10 around the cross-section contour). Each of these points is projected onto a cylindrical shape, in effect virtually rolling the flat preform around the thread axis. The conversion, connecting initial coordinate system $x, y, z$ to coordinates of the transformed geometry $\tilde{x}, \tilde{y}, \tilde{z}$, is implemented as follows: 


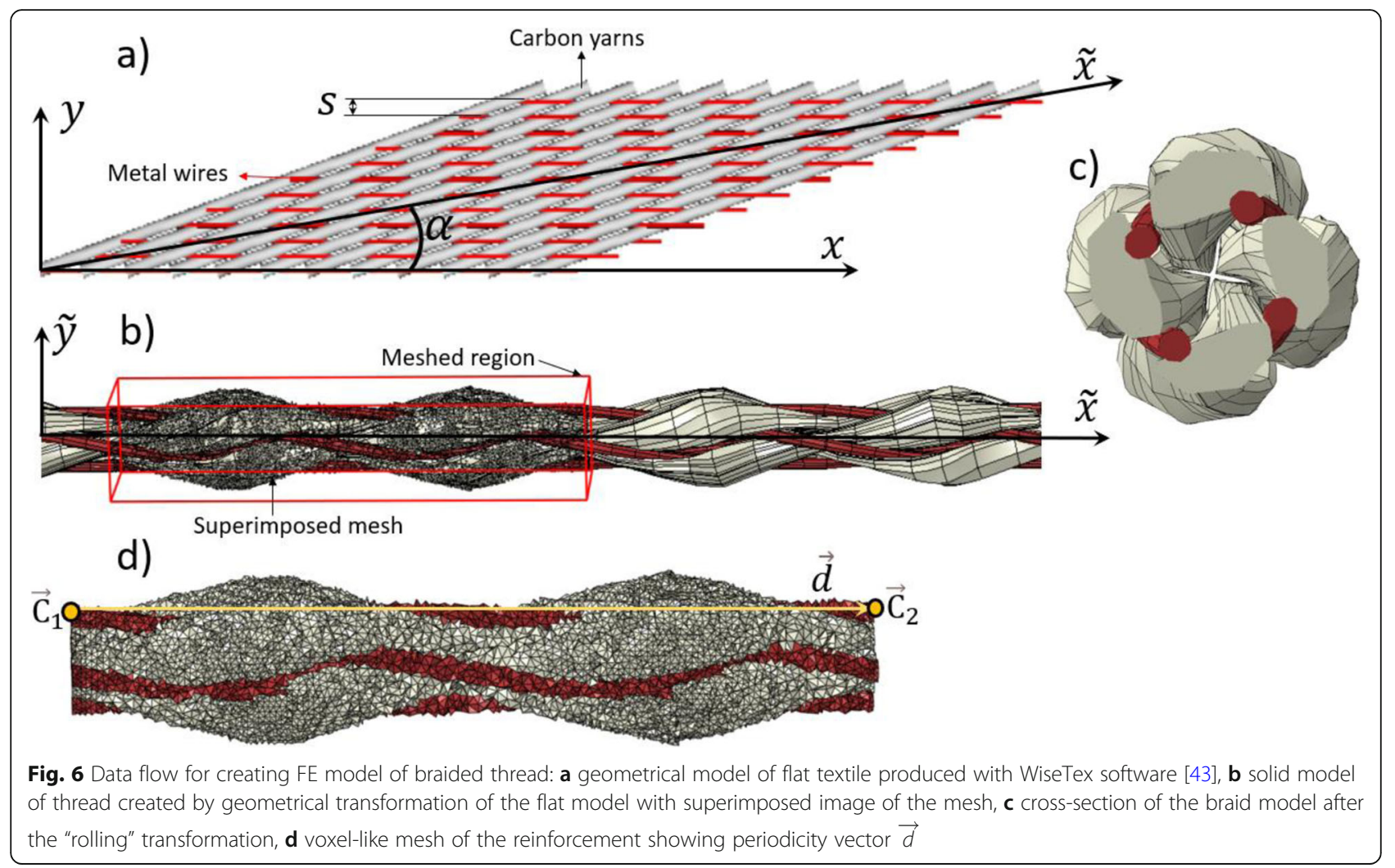

$$
\begin{aligned}
\bar{x} & =\left(x-x_{0}\right) \cos \alpha+\left(y-y_{0}\right) \sin \alpha \bar{y} \\
& =-\left(x-x_{0}\right) \sin \alpha+\left(y-y_{0}\right) \cos \alpha \bar{z}=z \\
r & =R+\bar{z} \theta=\frac{\bar{y}}{R} Z=\bar{x} \\
\hat{x} & =Z \hat{y}=r \sin \theta \hat{z}=r \cos \theta \\
\tilde{x} & =\hat{x} \cos \alpha-\hat{y} \sin \alpha \tilde{y}=\hat{x} \sin \alpha+\hat{y} \cos \alpha \tilde{z}=\hat{z}
\end{aligned}
$$

Where the $x$ axis is aligned with the direction of the metal wires in the unrolled representation of the braid geometry; $x_{0}, y_{0}$ - places the coordinate origin anywhere on the thread axis; $\tilde{x}$ - is aligned with the direction of braid, $\alpha$ is the braiding angle; $R=\frac{1}{2 \pi} \frac{N_{y} s}{\cos \alpha}$ is the radius of the braid; $s$ is the yarn spacing; $N_{y}=4$ is the number of braiding yarns of the same direction in the thread.

When considering a sufficiently large piece of the fabric, every 4th tow/wire of the same direction will eventually collapse onto the continuation of the correspondent preceding yarn and overlap with it at the offset distance of $\frac{N_{y} * s}{\cos \alpha}$. Hence, for a given pattern, braid angle and yarn spacing, the nominal radius of the thread is strictly defined.

Equation (1) reorients the coordinate system with the braid direction, Eq. (2) performs the rolling transformation of unrolled braid into the cylindrical geometry, Eqs. (3), (4) convert the cylindrical coordinates back to the cartesian ones and return to the original coordinate framework.

It is important to note that the transformation (2) introduces strong simplifying assumptions regarding the appearance of the "rolled" geometry. Any length within the braid nominal radius will decrease its size proportional to the radial distance from the nominal braid surface (transformed from the mid-plane of the fabric), whereas any length outside will correspondingly expand. This distorts the geometry and may lead to a contraction of some cross-sections below the mid-plane and expansion of the outer dimensions. In the first approximation, bearing in mind the calculation of the effective properties as a goal, this simplification deemed to be acceptable. Further geometry adjustments, as in [39], or postprocessing geometry in finite-element environment, as in [44], are possible and needed if the purpose of analysis shifts to high fidelity yarn-scale stress/current density calculations.

Once geometry is defined, the fibre orientation vectors $\vec{f}_{\text {new }}$, i.e. volume defined by neighbouring crosssections, is calculated using the Jacobian matrix J with elements $J_{i j}=\frac{\partial \tilde{x}_{i}}{\partial x_{j}}$ and the original fibre orientation vector $\vec{f}$ : 


$$
\vec{f}_{\text {new }}=\mathrm{J}^{-1} \vec{f}
$$

The same transformation applies to the vectors defining the orientations of every yarn cross-section in the model. Once the cross-section orientations, positions, and points defining their shapes are calculated, these are built through a series of sketches in a finite element environment (Dassault Systems Simulia Abaqus 2018) and lofted to make the 3D model of the braid. The resultant geometry is ready for meshing.

To model the electrical conduction problem, while avoiding excessive generation of mesh, a voxel-like approach is deployed. A block of the material is meshed with tetrahedral elements and then each of the elements are assigned material properties and orientations depending on where the centre of each element resides.

The suggested algorithm is rather fast - WiseTex generates the model of the unrolled fabric within seconds. The geometrical rolling operation, implemented in Python language included in Abaqus, calculates and lofts geometry within $30 \mathrm{~min}$ on a desktop of standard specification. The longest operation is assigning the material properties to individual elements in Abaqus which may take several hours.

A fragment of the unrolled braid, for illustration chosen to be 12 carbon yarns and 12 wires (though a smaller representative element could have been selected) is shown in Fig. 6a. It is modelled in WiseTex with the following parameters measured on manufactured materials: $s=0.36 \mathrm{~mm}, \alpha=10^{\circ}, R=0.23 \mathrm{~mm}$. Tow thickness in the model is restricted to the nominal thread diameter $R$, and the width by the spacing $s$. The main challenge is to balance these parameters between the requirements of a realistic inter-tow fibre volume fraction (i.e. to make the cross-section as large as practically possible) and, on the other hand, not making the braid architecture too tight as it makes the yarn midline path distorted and can cause a vital built-in Abaqus operation - solid lofting of yarns- to fail. For the case shown, the optimum was found to be a thickness/width of $0.18 / 0.33 \mathrm{~mm}$ for carbon tows resulting in $79 \%$ intra-tow fibre volume fraction. The cross section of each metal wire remains constant, modelled as a circle of diameter of $0.1 \mathrm{~mm}$. Fibre volume fraction can be further reduced if the solid geometry step is skipped.

The above example "rolled" geometry is shown in Fig. $6 \mathrm{~b}$. It can be seen that the representation of the thread is reasonably realistic, though the outer surfaces of the carbon tows expand beyond the tight geometry of the thread observed experimentally - Fig. 2. The crosssection of the braid shows that internal spacing of the model is filled rather well and leaves only a very small opening space, though no actual contact of yarns in the interior of the braid was explicitly modelled (Fig. 6c).
Figure $6 \mathrm{~d}$ illustrates selecting a representative element of the braid and meshing it. The linear size of elements, $0.03 \mathrm{~mm}$, was selected to be smaller than wire crosssection dimensions, so that connectivity of metal and percolation of electrical charge is preserved by the mesh. The voxel mesh also fills empty spaces free of tows and wires as well as covering any extra space on the tow periphery. These volumes are assigned zero conductivity and effectively do not contribute to the result calculation.

\section{Electrical conductivity of braids}

To calculate effective electrical conductivity of the braid, a linear electrical conduction problem statement is solved. Gauss's equation for electric field can be written as follows:

$$
\nabla \cdot(\sigma \nabla \phi)=0
$$

where $\phi$ is electric potential, $\sigma$ is electrical conductivity, $E=\sigma \nabla \phi$ is the electric field. Conservation of the electrical field requires boundary conditions on the edges of the domain under consideration. For the braid, we need to define the boundary conditions on the faces of the representative volume perpendicular to the thread direction. Thus we set periodic boundary conditions defining the difference in electric potential between the correspondent points on the opposite sides of the domain, which are separated by the periodicity vector $\vec{d}=\overrightarrow{\mathrm{C}}_{2}-\overrightarrow{\mathrm{C}}_{1}$ :

$$
\phi\left(\overrightarrow{\mathrm{C}}_{2}\right)-\phi\left(\overrightarrow{\mathrm{C}}_{1}\right)=\langle\nabla \phi\rangle d
$$

where points $\vec{C}_{2}$ and $\vec{C}_{1}$ represent any nodes on the two flat opposite faces of the thread volume which are perpendicular to the thread direction, $\langle\nabla \phi\rangle$ is the average electric potential gradient along the axis of the thread over the considered volume, $d$ is the length of the periodicity vector $\vec{d}$. The other faces of the volume are exposed to air and hence set to zero electric field.

When solving this equation for the representative volume of the braid, the generated solution results in a non-uniformly distributed electric field over the volume of the unit cell. Effective electrical conductivity of the braid $\sigma_{\text {eff }}$ can then be calculated as the average electric field in the braid direction $\left\langle\mathrm{E}_{1}\right\rangle$ normalised by the average electric potential gradient $\sigma_{\text {eff }}=\left\langle\mathrm{E}_{1}\right\rangle /\langle\nabla \phi\rangle$.

\section{Comparison of model and experimental results}

The result of this calculation will be dependent on the arbitrarily selected air volume enveloping the thread. Likewise, the experimental measurement of normalized resistance must be converted to conductivity by assuming a set braid diameter. In reality, this is difficult to measure: the apparent braid diameter will be dependent 
on pressure applied by calliper or on the location of measurements if conducted optically. Hence, to avoid this uncertainty, the results in both the experimental and numerical assessments of conductivity will be normalised with respect to the nominal braid diameter $R$.

$$
\tilde{\sigma}_{e f f}=\sigma_{e f f} \frac{A}{\pi R^{2}}
$$

where $\tilde{\sigma}_{\text {eff }}$ is the normalised effective conductivity, $A$ is the cross-sectional area of the considered braid volume. Similarly, the experimental measurements of conductivity will assume the nominal diameter of the thread $R$.

It is helpful to note that the formulation (6)-(8) is identical to the boundary value problem of heat transfer via thermal conductivity, where $\phi$ could be interpreted as temperature, $\sigma$ as thermal conductivity, and $\sigma \nabla \phi$ as thermal flux. Hence, rather than solving a reduced form of the Maxwell equations, an easier thermal solver can be deployed. Such solution has been implemented in Abaqus for this study.

Electrical conductivity of carbon tows within the thread is not precisely known and may be difficult to measure, particularly transverse conductivity. However, the analysis demonstrates that the precise values for carbon conductivity are not important. The electrical conductivity of copper wires is assumed equal to $5.96 \times 10^{7}$ $\mathrm{S} / \mathrm{m}$. The characteristic orthotropic conductivities of carbon tows are $1.00 \times 10^{4} \mathrm{~S} / \mathrm{m}$ in the longitudinal direction and $1.00 \mathrm{~S} / \mathrm{m}$ in the transverse direction. The latter is roughly in agreement with the through-thickness conductivity measured for non-tufted composite (characteristic figures for carbon composites can be found in [45, 46]). Based on these input values, the effective electrical conductivity is calculated as $1.0282 \times 10^{7} \mathrm{~S} / \mathrm{m}-$ Table 2 . When doubling longitudinal conductivity of carbon tows, the effective value changes to $1.0284 \times 10^{7} \mathrm{~S} / \mathrm{m}$. Alternatively, when increasing the transverse conductivity of tows by two orders of magnitude to $100 \mathrm{~S} / \mathrm{m}$ (corresponding to some extreme values reported in literature for nano-additive enhanced carbon composites), the effective conductivity does not make an appreciable impact beyond numerical noise. This shows that electrical conductivity of carbon is so low compared to metal that the effective conductivity is solely defined by the crimp and properties of metal wires. Carbon fibres in this context play only the role of spacers affecting the trajectories of the metal.

Comparing the obtained values with the conductivity of four isolated wires encircled by the effective area with the nominal thread radius $R\left(1.1267 \times 10^{7} \mathrm{~S} / \mathrm{m}\right)$, we see that wire crimp decreases the conductivity by approximately $9 \%$. The crimp, calculated WiseTex model, is estimated to be below $0.1 \%$ (as opposed to $3.2 \%$ of crimp of braiding yarns). Spinning the wire within a tubular braid adds to the crimp and is estimated to be $1.5 \%$. Experimental measurements of resistance, discussed in the previous section, give a conductivity of $0.601 \times 10^{7} \mathrm{~S} / \mathrm{m}$ which is roughly half of the predicted value. This could mean that the electrode clamps were not in contact with all four wires within a braid or indicate damage to the wires from either the braiding or tufting process. The measurement of conductivity from the example braid with minimum normalized resistance prior to testing may confirm this hypothesis - a mean value of $(1.50 \pm$ $0.15) \times 10^{7} \mathrm{~S} / \mathrm{m}$ is found, which is well in agreement with predictions of the model. The experimental measurement of conductivity at the end of a tensile test, following braid failure, is consistent with only one wire remaining.

Examining the local conductivity of blocks cut from the panel (10 by $10 \mathrm{~mm}$ in the plane of the laminate) shows much lower conductivity that could be expected from the assessment of the individual tuft. The maximum conductivity in those measurements did not exceed $100 \mathrm{~S} / \mathrm{m}$. On the one hand, this is two orders of magnitude higher than the conductivity of the reference untufted panel. On the other hand, the value is more

Table 2 Electrical conductivities of hybrid braided threads and composites tufted with them

\begin{tabular}{|c|c|}
\hline Electrical conductivity & $\mathrm{S} / \mathrm{m}$ \\
\hline Conductivity of copper wire & $5.96 \times 10^{7}$ \\
\hline Measured on straightened threads ${ }^{a}$ & $(0.60 \pm 0.30) \times 10^{7}$ \\
\hline Conductivity of most conductive threads averaged over time ${ }^{a}$ & $(1.50 \pm 0.15) \times 10^{7}$ \\
\hline Calculated for hybrid thread ${ }^{a}, b$ & $1.03 \times 10^{7}$ \\
\hline Four straight copper wires in a cylinder of radius $R$ & $1.13 \times 10^{7}$ \\
\hline Through-thickness of non-tufted carbon-epoxy ${ }^{c}$ & $0.74 \pm 0.07$ \\
\hline Through-thickness of tufted, minimum ${ }^{c}$ & 0.93 \\
\hline Through-thickness of tufted, maximum ${ }^{c}$ & 98.89 \\
\hline
\end{tabular}

\footnotetext{
a at nominal radius of $R=0.23 \mathrm{~mm}$,

$\mathrm{b}$ input longitudinal/transverse conductivity of carbon of $10^{4} / 1 \mathrm{~S} / \mathrm{m}$

c measured at cut blocks of $10 \times 10 \mathrm{~mm}$ in the plane of the laminate
} 
than two orders of magnitude lower than if the thread was ideally aligned and span the block in entirety from top to bottom. This indicates that there is no full through-thickness percolation in the measured sample blocks and complex tuft paths does not necessarily span from one measurement plane to the other.

\section{Conclusions and discussion}

The results obtained in this study demonstrate the potential of using hybrid multi-material braids to increase through thickness electrical conductivity in carbon fibre/ epoxy composites. It has been shown that the braid pattern chosen confers both high conductivity, courtesy of the metal filaments, and reliable structural integrity. In the subsequent process of tufting, a part of that functionality may be lost due to the breakage of some metal wires and added crimp of the thread. Yet, most of the features of the braided architecture are preserved, with the increased local conductivity of a tufted panel compared to an untufted benchmark demonstrating the improvement in through-thickness conductivity. This is achieved in addition to the structural benefits provided by through-thickness reinforcement and using a well understood manufacturing technique. Further optimisation of the composite properties may be achieved through tailoring parameters of the tufting process to the mechanical properties of the hybrid threads.

The new way of integrating conductive elements opens an avenue for many applications where through-thickness functional performance is essential. There is a clear potential for sensing applications and a natural system for structural health monitoring via electrical resistance. Other applications may include heating by electromagnetic induction, which has been demonstrated for curing adhesive bonds [43]. It is hypothesised that elevated electrical conductivity could help formation of through-thickness loops for eddy current circulation and hence make the material more susceptible to heat via oscillating magnetic fields, perhaps tufting braids only in an area where higher temperatures are needed or using braids to add conductivity to glass fibre, aramid or natural fibre panels. The architecture of tufts and tufted pattern may be essential here. These hypotheses require an experimental investigation, which is the subject of a separate study.

Conductive additions such as mesh or foil can be used for lighting strike protection, current carrying or electromagnetic interference shielding. These inclusions may be detrimental to the structural properties of the composite, whereas tufting delivers through thickness reinforcement. It is therefore worth considering whether applications of this nature could be achieved by using tufted braids instead.

The paper presented a simple, fast modelling tool highlighting some of the essential features of the braid and demonstrating a reasonably realistic representation of its internal geometry. The resulting simulation is used to calculate electrical conductivity, showing that for this braid model sophisticated finite-element modelling is not needed. The three orders of magnitude contrast between conductivities of tows and wires and the linear geometry of the thread makes predictions of conductivity insensitive to the fine features of internal geometry and hence more straightforward. The geometrical model of the 'unrolled' braid fabric and simple transformation to construct the 'rolled' braid is sufficient for a good insight into the characteristic behaviour of the braid and the relation between braid geometry and its functional characteristics. Looking to the future, such model could serve as a good precursor to mechanical modelling of the braid behaviour where the interaction between carbon and metal may play a significant role.

\section{Acknowledgements}

Authors would like to thank Michael Elkington for useful discussions and Yusuf Mahadik for support with examination of the studied materials. CT scanning has been conducted on facilities of the National Composites Centre, Bristol.

\section{Authors' contributions \\ CO'K led trials on creating multifunctional braided threads and conducted their experimental characterisation. CO'K and LP tufted dry preforms with hybrid threads and manufactured multifunctional composites. CO'K and JC examined electrical properties of the panels and their morphology. IKP and GA advised on various aspects of testing and manufacturing. DI created modelling tool for the analysis of the hybrid threads and coordinated work. All authors read and approved the final manuscript.}

\section{Funding}

The work has been supported by the EPSRC through the Future Composites Manufacturing Research Hub [EP/ P006701/1] as part of its Core Project "Manufacturing for structural applications of multifunctional composites".

Availability of data and materials

All data to support the conclusions reached are included in full in this article. Supplementary data is available at https://doi.org/10.5523/bris.

28mdwiikm2tw31z25aaga7p3za.

\section{Competing interests}

The authors declare that they have no competing interests.

\section{Author details}

'Bristol Composites Institute http://www.bristol.ac.uk/composites/ACCIS), University of Bristol, University Walk, Bristol BS8 1TR, UK. ${ }^{2}$ COMAC Shanghai Aircraft Design \& Research Institute, 5188 Jinke Road, Zhangjiang, Shanghai, China.

Received: 9 December 2020 Accepted: 27 January 2021

Published online: 17 February 2021

\section{References}

1. C. González, J.J. Vilatela, J.M. Molina-Aldareguía, C.S. Lopes, J. LLorca, Structural composites for multifunctional applications: Current challenges and future trends. Prog. Mater. Sci. 89, 194-251 (2017). https://doi.org/10. 1016/.jpmatsci.2017.04.005

2. A.M. Karlsson, M.O. Adeoye, in Encyclopedia of Complexity and Systems Science. Composites, Multifunctional (Springer, New York, 2009), pp. 12831302

3. B. Gu, H. Zhang, B. Wang, S. Zhang, X. Feng, Fracture toughness of laminates reinforced by piezoelectric z-pins. Theor. Appl. Fract. Mech. 77, 35-40 (2015). https://doi.org/10.1016/j.tafmec.2015.01.007 
4. B. Alemour, O. Badran, and M. R. Hassan, "A review of using conductive composite materials in solving lightening strike and ice accumulation problems in aviation," J. Aerosp. Technol. Manag., 2019, doi: https://doi.org/ 10.5028/jatm.v11.1022

5. M. Gagné, D. Therriault, Lightning strike protection of composites. Prog. Aerosp. Sci. 64, 1-16 (2014). https://doi.org/10.1016/j.paerosci.2013.07.002

6. M.G. Callens, L. Gorbatikh, I. Verpoest, Ductile steel fibre composites with brittle and ductile matrices. Compos. Part A 61, 235-244 (2014). https://doi. org/10.1016/j.compositesa.2014.02.006

7. U.P. Breuer, S. Schmeer, in Multifunctionality of Polymer Composites: Challenges and New Solutions, ed. by K. Friedrich, U. Breuer. Carbon and metal-fiberreinforced airframe structures (Elsevier Inc., New York, 2015), pp. 435-447

8. H. Kawakami, P. Feraboli, Lightning strike damage resistance and tolerance of scarf-repaired mesh-protected carbon fiber composites. Compos. Part A Appl. Sci. Manuf. 42(9), 1247-1262 (2011). https://doi.org/10.1016/j. compositesa.2011.05.007

9. C. O'Brien, A.E. Zaghi, Mechanical characteristics of hybrid composites with $\pm 45^{\circ}$ glass and $0^{\circ} / 90^{\circ}$ stainless steel fibers. Materials (Basel) 11, 1355-1372 (2018). https://doi.org/10.3390/ma11081355

10. A.K. Mcbride, S.L. Turek, A.E. Zaghi, K.A. Burke, Mechanical behavior of hybrid glass / steel fiber reinforced epoxy composites. Polymers (Basel) 9, 1-16 (2017). https://doi.org/10.3390/polym9040151

11. K. Friedrich, in Multifunctionality of Polymer Composites: Challenges and New Solutions, no. 1, ed. by K. Friedrich, U. Breuer. Routes for achieving multifunctionality in reinforced polymers and composite structures (Elsevier Inc., New York, 2015), pp. 3-41

12. N.D. Alexopoulos, C. Bartholome, P. Poulin, Z. Marioli-Riga, Structural health monitoring of glass fiber reinforced composites using embedded carbon nanotube (CNT) fibers. Compos. Sci. Technol. 70(2), 260-271 (2010). https:// doi.org/10.1016/j.compscitech.2009.10.017

13. L. Nicolais, G. Carotenuto, Nanocomposites with tailored optical properties. Editor(s): Klaus Friedrich, Ulf Breuer, Multifunctionality of Polymer Composites, Elsevier, Oxford, UK, 28, 842-857, (2015) https://doi.org/10.1016/ B978-0-323-26434-1.00028-3.

14. L. Christodoulou, J.D. Venables, Multifunctional material systems: The first generation. J. Miner. Met. Mater. Soc. 55(12), 39-45 (2003). https://doi.org/ 10.1007/s11837-003-0008-z

15. R.F. Gibson, A review of recent research on mechanics of multifunctional composite materials and structures. Compos. Struct. 92(12), 2793-2810 (2010). https://doi.org/10.1016/j.compstruct.2010.05.003

16. K. Salonitis, J. Pandremenos, J. Paralikas, G. Chryssolouris, Multifunctional materials: Engineering applications and processing challenges. Int. J. Adv. Manuf. Technol. 49(5-8), 803-826 (2010). https://doi.org/10.1007/s00170009-2428-6

17. A.D.B.L. Ferreira, P.R.O. Nóvoa, A.T. Marques, Multifunctional material systems: A state-of-the-art review. Compos. Struct. 151, 3-35 (2016). https://doi.org/ 10.1016/j.compstruct.2016.01.028

18. K.K. Sairajan, G.S. Aglietti, K.M. Mani, A review of multifunctional structure technology for aerospace applications. Acta Astronaut. 120, 30-42 (2016). https://doi.org/10.1016/j.actaastro.2015.11.024

19. K.J. Narayana, R. Gupta Burela, A review of recent research on multifunctional composite materials and structures with their applications. Mater. Today Proc. 5(2), 5580-5590 (2018). https://doi.org/10.1016/j.matpr.2017.12.149

20. C. Osmiani, G. Mohamed, J.W.G. Treiber, G. Allegri, I.K. Partridge, Exploring the influence of micro-structure on the mechanical properties and crack bridging mechanisms of fibrous tufts. Compos. Part A Appl. Sci. Manuf. 91 409-419 (2016). https://doi.org/10.1016/j.compositesa.2016.08.008

21. D. M. Lombetti, "Tufting of complex composite structures," PhD Thesis, Cranfield Univeristy 2015

22. I. Gnaba, X. Legrand, P. Wang, D. Soulat, Literature review of tufted reinforcement for composite structures. IOP Conf. Ser. Mater. Sci. Eng. 254(4), 042011 (2017). https://doi.org/10.1088/1757-899X/254/4/042011

23. I. Gnaba, P. Wang, X. Legrand, D. Soulat, Manufacturing and characterization of tufted preform with complex shape. Adv. Aircr. Spacecr. Sci. 6(2), 105116 (2019). https://doi.org/10.12989/aas.2019.6.2.105

24. D.M. Lombetti, A.A. Skordos, Lightning strike and delamination performance of metal tufted carbon composites. Compos. Struct. 209, 694-699 (2019). https://doi.org/10.1016/j.compstruct.2018.11.005

25. M. Scott, G. Dell'Anno, H. Clegg, Effect of process parameters on the geometry of composite parts reinforced by through-the-thickness tufting. Appl. Compos. Mater., 1-12 (2018). https://doi.org/10.1007/s10443-018-9710-4
26. G. Dell'Anno, "Effect of tufting on the mechanical behaviour of carbon fabric/epoxy composites," PhD Thesis, Cranfield University 2007

27. J. W. G. Treiber, "Performance of tufted carbon fibre/epoxy composites," PhD Thesis, Cranfield University, 2011

28. C. Hui, P. Wang, X. Legrand, Improvement of tufting mechanism during the advanced 3-dimensional tufted composites manufacturing: To the optimisation of tufting threads degradation. Compos. Struct. 220, 423-430 (Jul. 2019). https://doi.org/10.1016/j.compstruct.2019.04.019

29. A.T. Martins, Z. Aboura, W. Harizi, A. Laksimi, K. Hamdi, Structural health monitoring by the piezoresistive response of tufted reinforcements in sandwich composite panels. Compos. Struct. 210(July 2018), 109-117 (2019). https://doi.org/10.1016/j.compstruct.2018.11.032

30. A.T.T. Martins, Z. Aboura, W. Harizi, A. Laksimi, K. Khellil, Structural health monitoring for GFRP composite by the piezoresistive response in the tufted reinforcements. Compos. Struct. 209, 103-111 (2019). https://doi.org/10. 1016/j.compstruct.2018.10.091

31. E. Chehura, G. Dell'Anno, T. Huet, S. Staines, S.W. James, I.K. Partridge, R.P. Tatam, On-line monitoring of multi-component strain development in a tufting needle using optical fibre Bragg grating sensors. Smart Mater. Struct. 23, 1-9 (2014). https://doi.org/10.1088/0964-1726/23/7/075001

32. G. Dell'Anno, J.W.G. Treiber, I.K. Partridge, Manufacturing of composite parts reinforced through-thickness by tufting. Robot. Comput. Integr. Manuf. 37, 262-272 (2016). https://doi.org/10.1016/j.rcim.2015.04.004

33. C. O'Keeffe, G. Allegri, I.K. Partridge, in SMS 2018 Smart Materials and Surfaces, Venice. Hybrid Multimaterial Microbraids for Through-Thickness Multifunctionality (2018)

34. C. O'Keeffe, G. Allegri, I.K. Partridge, in 1st European Conference on Crashworthiness of Composite Structures - ECCCS-1, Belfast. Hybrid multimatrials microbraids for through-thickness multi-functionality (2019)

35. J.P. Carey, Handbook of advances in braided composite materials: theory, production, testing and applications Duxford: Elsevier Science (2016)

36. D.S. Ivanov, F. Baudry, B. Van Den Broucke, S.V. Lomov, H. Xie, I. Verpoest, Failure analysis of triaxial braided composite. Compos. Sci. Technol. 69(9) (2009). https://doi.org/10.1016/j.compscitech.2008.09.013

37. A.K. Pickett, J. Sirtautas, A. Erber, Braiding simulation and prediction of mechanical properties. Appl. Compos. Mater. 16(6), 345-364 (2009). https:// doi.org/10.1007/s10443-009-9102-x

38. I. Verpoest, S.V. Lomov, Virtual textile composites software WiseTex: Integration with micro-mechanical, permeability and structural analysis. Compos. Sci. Technol. 65, 2563-2574 (2005). https://doi.org/10.1016/j.compscitech.2005.05.031

39. S.V. Lomov, I. Verpoest, J. Cichosz, C. Hahn, D.S. Ivanov, B. Verleye, Mesolevel textile composites simulations: Open data exchange and scripting. J. Compos. Mater. 48(5), 621-637 (2014). https://doi.org/10.1177/ 0021998313476327

40. British Standards Institution, "BS EN ISO 2062:2009. Textiles — Yarns from packages - Determination of single-end breaking force and elongation at break using constant rate of extension." 2009

41. British Standards Institution, "BS 7658-2: 1993, ISO 10120:1991. Carbon fibre — Part 2: Method for determination of linear density," 1993

42. S.C. Brown, C. Robert, V. Koutsos, D. Ray, Methods of modifying throughthickness electrical conductivity of CFRP for use in structural health monitoring and its effect on mechanical properties. Compos. Part A 133(March), 105885 (2020). https://doi.org/10.1016/j.compositesa.2020.105885

43. T. Bayerl, M. Duhovic, P. Mitschang, D. Bhattacharyya, The heating of polymer composites by electromagnetic induction - a review. Compos. Part A Appl. Sci. Manuf. 57(2014), 27-40 (2014). https://doi.org/10.1016/j.compositesa.2013.10.024

44. S.V. Lomov, D.S. Ivanov, I. Verpoest, M. Zako, T. Kurashiki, H. Nakai, S. Hirosawa, Meso-FE modelling of textile composites: Road map, data flow and algorithms. Compos. Sci. Technol. 67(9), 1870-1891 (2007). https://doi. org/10.1016/j.compscitech.2006.10.017

45. R. Rudolf, P. Mitschang, M. Neitzel, Induction heating of continuous carbonfibre-reinforced thermoplastics. Compos. Part A Appl. Sci. Manuf. 31(11), 1191-1202 (2000). https://doi.org/10.1016/S1359-835X(00)00094-4

46. J.B. Park, T.K. Hwang, H.G. Kim, Y.D. Doh, Experimental and numerical study of the electrical anisotropy in unidirectional carbon-fiber-reinforced polymer composites. Smart Mater. Struct. 16(1), 57-66 (2007). https://doi.org/10. 1088/0964-1726/16/1/006

\section{Publisher's Note}

Springer Nature remains neutral with regard to jurisdictional claims in published maps and institutional affiliations. 